\title{
COBORDISM OPERATIONS AND HOPF ALGEBRAS
}

\author{
BY \\ P. S. LANDWEBER
}

1. Introduction. This paper is concerned with the stable operations of the unoriented, complex and symplectic cobordism theories $H^{*}(\quad ; M O), H^{*}(; M U)$ and $H^{*}(\quad ; \boldsymbol{M S p})$ on the category of finite $C W$-pairs (see [5], [6], [15]). The starting point is Theorem (3.1): the algebra (under composition) $\mathscr{A}^{*}(G)$ of stable operations of $H^{*}(; M G)$ is additively isomorphic to the algebra $\mathscr{C}^{*}(G)$ of stable characteristic classes of $G$-bundles with values in $G$-cobordism; this is true not only for $\boldsymbol{O}, \boldsymbol{U}$ and $\boldsymbol{S p}$ but also for the other stable groups which give rise to cobordism theories. For $\boldsymbol{G}=\boldsymbol{O}, \boldsymbol{U}$ or $\boldsymbol{S p}$, Conner and Floyd introduced in [5], [7] characteristic classes $E_{i}(\xi) \in H^{d i}(X ; M G)$ for $G$-bundles $\xi \rightarrow X$ (see $\left.\S 4\right)$; here $d=1,2$ or 4 for $G=\boldsymbol{O}, \boldsymbol{U}$ or $S p$, resp. The $E_{\mathfrak{i}}$ generate a polynomial subalgebra $C^{*}(G)$ of $\mathscr{C}^{*}(G)$, to which there corresponds a submodule $A^{*}(G)=\sum A^{i}(G)(i \geqq 0)$ of $\mathscr{A}^{*}(G)$.

It is shown in $\S 5$ that $A^{*}(G)$ is a graded Hopf algebra, over the integers for $G=U$ or $S p$ and over $Z_{2}$ in the unoriented case. In particular $A^{*}(G)$ is closed under the composition of operations; this product is studied in $\$ 6$ with the help of the dual Hopf algebra $A_{*}(G)$. In $\$ 7$ minimal generating sets are found for $A^{*}(G) \otimes Z_{p}$ ( $G=\boldsymbol{U}$ or $S p, p$ a prime) and $A^{*}(\boldsymbol{O})$, and in the following section these Hopf algebras are shown to contain quotients of the Steenrod algebras $\mathscr{S}^{*}(p)$. For example, $A^{*}(\boldsymbol{U}) \otimes Z_{p}$ contains an isomorphic copy of $\mathscr{S}^{*}(p) /\left(\beta_{p}\right)$ with $\beta_{p}$ the Bockstein and $A^{*}(\boldsymbol{O})$ contains a copy of the mod 2 Steenrod algebra $\mathscr{S}^{*}(2)$.

In the final section some comments are made concerning the action

$$
A^{i}(G) \otimes \Omega_{n}^{G} \rightarrow \Omega_{n-i}^{G}
$$

of $A^{*}(G)$ on the $G$-bordism ring $\Omega_{*}^{G}$, obtained via the identification of $\Omega_{*}^{G}$ with the coefficient ring $H^{*}(p t ; M G)$. Although this action provided the motivation for the present study, it remains quite obscure even in the unoriented case.

2. Notation and preliminary results. Let $G$ be $\boldsymbol{O}, \boldsymbol{U}$ or $\boldsymbol{S p}$. When dealing with cohomology, $Z_{2}$ will be understood as coefficients if $\boldsymbol{G}=\boldsymbol{O}$ and the integers in the other cases. We assume familiarity with the Stiefel-Whitney classes $w_{i}(\xi)$ of real bundles and the Chern classes $c_{i}(\xi)$ of complex bundles. For $\xi$ a right quaternionic bundle, which may also be regarded as a complex bundle, the symplectic Pontrjagin class $p_{i}(\xi)$ is by definition $(-1)^{i} c_{2 \imath}(\xi)$; these satisfy the Whitney sum formula (see $[4, \S 9])$. Thus in all cases, for $\xi \rightarrow X$ a $G$-bundle over a finite $C W$-complex there are characteristic classes $e_{i}(\xi) \in H^{d i}(X)$, with $d=1,2$ or 4 for $G=\boldsymbol{O}, \boldsymbol{U}$ or $S p$, resp.

Presented to the Society, September 1, 1965 under the title Cobordism operations; received by the editors December 21, 1965 and, in revised form, October 14, 1966. 
For any stable group $G, B G(r)$ can be approximated by finite subcomplexes. If $G=\boldsymbol{O}, \boldsymbol{U}$ or $\boldsymbol{S p}$ we may make this explicit as follows. Let $K$ denote the field $\boldsymbol{R}, \boldsymbol{C}$ or $\boldsymbol{H}$ corresponding to the choice of $G$, and denote by $G_{r, s}(K)$ the Grassmann manifold of $r$-planes in the (right) vector space $K^{r+s}$. Thus $G_{r, \infty}(K)=B G(r)$ is the classifying space of $G(r)$, and $G_{r, s}(K)$ approximates $B G(r)$ as $s \rightarrow \infty$. In addition to the standard $r$-plane bundle $\xi_{r, s}$ over $G_{r, s}(K)$, there is an $s$-plane bundle $\eta_{r, s}$, with $\xi \oplus \eta$ trivial. Let $e_{i}=e_{i}(\xi) \in H^{d i}\left(G_{r, s}(K)\right)$ and $\bar{e}_{i}=e_{i}(\eta)$ be the characteristic classes of $\xi$ and $\eta$. The Whitney sum formula implies that

$$
\sum_{i+j=k} e_{i} \bar{e}_{j}=0 \quad(0<k \leqq r+s) .
$$

In fact, these generate all relations on the $e_{i}$ and $\bar{e}_{j}$ (see [2], [3]):

(2.1) $H^{*}\left(G_{r, s}(K)\right)$ is generated by $e_{1}, \ldots, e_{r}$ and $\bar{e}_{1}, \ldots, \bar{e}_{s}$ subject to the above relations.

Moreover, $H^{*}\left(G_{r, s}(K)\right)$ is generated by $e_{1}, \ldots, e_{r}$ since the $\bar{e}_{j}$ can be expressed in terms of the $e_{i} . H^{*}\left(G_{r, s}(K)\right)$ is a polynomial algebra on $e_{1}, \ldots, e_{r}$ up to dimension $d s$.

Several points of cobordism theory will now be reviewed; greater detail may be found in [5], [6], [7]. We maintain the convention throughout that $d=1,2$ or 4 according as $G=\boldsymbol{O}, \boldsymbol{U}$ or $\boldsymbol{S p}$. For $(X, A)$ a finite $C W$-pair,

$$
H^{n}(X, A ; M G)=\left[S^{d k-n}(X \mid A), M G(k)\right]
$$

for $k$ large. Let $\xi \rightarrow X$ be a $G(n)$-bundle over a finite $C W$-complex. There is a classifying map $X \rightarrow B G(n)$, which gives rise to a map $M(\xi) \rightarrow M G(n)$ of Thom spaces, and so to an element $u_{\xi}$ of $\bar{H}^{\text {dn }}(M(\xi) ; M G)$, the $G$-cobordism Thom class of $\xi$. It follows from Dold's theorem [8] that there is a Thom isomorphism

$$
\phi: H^{k}(X ; M G) \rightarrow \bar{H}^{k+d n}(M(\xi) ; M G)
$$

given by $\phi(x)=u_{\xi} \cdot x$.

There is a natural transformation $\mu: H^{*}(; M G) \rightarrow H^{*}(\quad)$ to cohomology, mod 2 in the unoriented case and integral otherwise. Various forms of the following result may be found in [5], [6, §18].

(2.2) Let $(X, A)$ be a finite $C W$-pair, with $H^{*}(X, A)$ free abelian if $G=U$ or $S p$. Suppose that $K^{*} \subset H^{*}(X, A ; M G)$ is mapped isomorphically onto $H^{*}(X, A)$ by $\mu$. Then $\Omega_{G}^{*} \otimes K^{*} \rightarrow H^{*}(X, A ; M G)$ is an isomorphism of $\Omega_{G}^{*}$-modules.

Here $\Omega_{G}^{*}=H^{*}(p t ; M G)$ is the coefficient ring, and the conclusion states that $H^{*}(X, A ; M G)$ is a free $\Omega_{G}^{*}$-module.

Let $M^{*}=\sum_{i \leq 0} M^{i}$ and $N^{*}=\sum_{j \geqq 0} N^{j}$ be graded abelian groups. The completed tensor product of $M^{*}$ and $N^{*}$ is the graded group $M^{*} \widehat{\otimes} N^{*}=\sum_{k=-\infty}^{\infty}\left(M^{*} \widehat{\otimes} N^{*}\right)^{k}$, with

$$
\left(M^{*} \widehat{\otimes} N^{*}\right)^{k}=\prod_{i+j=k} M^{i} \otimes N^{j} .
$$


Thus if $M^{i}=0$ for $i<i_{0}$ or $N^{j}=0$ for $j>j_{0}$, then $M^{*} \hat{\otimes} N^{*}$ is simply the graded tensor product $M^{*} \otimes N^{*}$. Elements of $\left(M^{*} \widehat{\otimes} N^{*}\right)^{k}$ will be written as infinite sums $\sum m_{i} \otimes n_{i}$, with the understanding that the degrees of the $m_{\mathfrak{i}}$ tend to $+\infty$ (and those of the $n_{t}$ to $-\infty$ ). The following easily proved result will be used in $\$ 4$.

(2.3) Let $M^{*}=\sum_{i \leqq 0} M^{i}$ and $N^{*}=\sum_{j \geqq 0} N^{j}$ be graded abelian groups, with the $N^{*}$ forming an inverse system. If all the $M^{i}$ are free abelian of finite rank, then

$$
\lim \operatorname{inv}\left(M^{*} \hat{\otimes} N_{\alpha}^{*}\right) \approx M^{*} \hat{\otimes}\left(\lim \operatorname{inv} N_{\alpha}^{*}\right) .
$$

3. Cobordism operations and characteristic classes. Throughout this section $G$ will denote a fixed stable group such as $\boldsymbol{O}, \boldsymbol{U}, \boldsymbol{S p}$ or one of the other groups giving rise to a cobordism theory. Thus there is a Thom spectrum $M G$ and the notion of $G$-bundle.

By a $G$-cobordism operation $\theta$ of degree $i$ is meant a family of linear mappings

$$
\theta: H^{k}(X, A ; M G) \rightarrow H^{k+i}(X, A ; M G),
$$

defined for all $C W$-pairs, which commutes with induced maps and suspensions. The totality of these operations is denoted $\mathscr{A}^{i}(G)$. Thus $\mathscr{A}^{*}(G)=\sum_{i=-\infty}^{\infty} \mathscr{A}^{i}(G)$ is a graded algebra with addition "pointwise" and multiplication given by the composition of operations.

Since $H^{*}(X, A ; M G)$ is a left graded module over the coefficient ring

$$
\Omega_{G}^{*}=H^{*}(p t ; M G),
$$

$\Omega_{G}^{*}$ may be regarded as a subalgebra of $\mathscr{A}^{*}(G)$.

A $G$-cobordism characteristic class $\gamma$ of degree $i$ is a functor which assigns to each $G$-bundle $\xi \rightarrow X$ over a finite $C W$-complex a cobordism class $\gamma(\xi) \in H^{i}(X ; M G)$, and which is natural with respect to $G$-bundle maps and is stable: $\gamma(\xi \oplus 1)=\gamma(\xi)$. Let $\mathscr{C}^{i}(G)$ denote these characteristic classes, and put $\mathscr{C}^{*}(G)=\sum_{i=-\infty}^{\infty} \mathscr{C}^{i}(G)$. Thus $\mathscr{C}^{*}(G)$ is a graded algebra with pointwise operations. For $n \in \Omega_{G}^{i}$,

$$
\xi \rightarrow n \cdot 1 \in H^{i}(X ; M G)
$$

is an element of $\mathscr{C}^{i}(G)$, so $\Omega_{G}^{*}$ is trivially a subalgebra of $\mathscr{C}^{*}(G)$.

The rest of this section is devoted to showing that $\mathscr{A}^{*}(G)$ and $\mathscr{C}^{*}(G)$ are isomorphic additively. Let $\Psi: \mathscr{A}^{*}(G) \rightarrow \mathscr{C}^{*}(G)$ be defined in the following familiar manner. For $\theta \in \mathscr{A}^{*}(G)$ and $\xi \rightarrow X$ a $G$-bundle, put

$$
\Psi(\theta)(\xi)=\phi^{-1} \theta \phi(1),
$$

where $\phi$ is the $G$-cobordism Thom isomorphism of $\xi$.

(3.1) THEOREM. $\Psi: \mathscr{A}^{*}(G) \rightarrow \mathscr{C}^{*}(G)$ is an isomorphism of graded abelian groups.

Proof. It suffices to define an inverse $\Phi$ for $\Psi$, Let $\gamma \in \mathscr{C}^{*}(G)$ be given. If $u_{\xi}$ is the $G$-cobordism Thom class of a $G$-bundle $\xi$, put $\Phi(\gamma) \cdot u_{\xi}=\phi(\gamma(\xi))$, to assure that $\Phi$ be an inverse for $\Psi$. If now $\alpha \in H^{n}(X, A ; M G)$ is any cobordism class, represent $\alpha$ by a map $f: S^{d k-n}(X \mid A) \rightarrow M G(k)$, and assume $M G(k)$ replaced by a finite 
approximation. Thus the suspension $\sigma^{d k-n}(\alpha)$ equals $f^{*}\left(u_{k}\right)$, with $u_{k}$ the $G$ cobordism Thom class of the (finite) universal $G$-bundle over $B G(k)$. Then put

$$
\Phi(\gamma) \cdot \alpha=\left(\sigma^{d k-n}\right)^{-1} f^{*}\left(\Phi(\gamma) \cdot u_{k}\right) .
$$

It is easily checked that $\Phi$ is well defined, linear and degree preserving, and it was constructed to be an inverse for $\Psi$. This completes the proof.

More generally, if $\mathscr{H}^{*}$ is a generalized cohomology theory with a functorial Thom isomorphism for $G$-bundles, then there is an analogous correspondence between the stable operations $H^{*}(\quad ; M G) \rightarrow \mathscr{H}^{*}(\quad)$ and the stable $\mathscr{H}^{*}$-characteristic classes of $G$-bundles. Notice that a $G$-cobordism operation is determined by its values on the Thom classes of $G$-bundles, in fact by its values on the Thom classes of the finite universal $G$-bundles.

4. Construction of characteristic classes. Let $G=O, U$ or $S p$ and $d=1,2$ or 4 accordingly. For $\xi \rightarrow X$ a $G(1)$-bundle over a finite complex, define $E_{1}(\xi)$ in $H^{d}(X ; M G)$ as follows. Choose a classifying map $X \rightarrow B G(1)$ and compose it with the inclusion $B G(1) \subset M G(1)$ to obtain a map $X \rightarrow M G(1)$, which gives rise to an element $E_{1}(\xi)$ of $H^{d}(X ; M G)$. It is easily seen that $\mu\left(E_{1}(\xi)\right)=e_{1}(\xi) \in H^{d}(X)$, with $\mu$ the natural transformation from $G$-cobordism to cohomology. Thus the hypotheses of $[7,(7.5)]$ and its analogues for the real and complex case are satisfied, so we obtain

(4.1) Theorem. For $G=\boldsymbol{O}, U$ or $S p$, there are unique $G$-cobordism classes $E_{i} \in \mathscr{C}^{d i}(G)$ with $E_{0}=1$ satisfying

(1) $E_{k}(\xi \oplus \eta)=\sum_{i+j=k} E_{i}(\xi) E_{j}(\eta)$;

(2) for $\xi$ a $G(1)$-bundle, $E_{1}(\xi)$ is defined as above and $E_{i}(\xi)=0, i>1$. Moreover, $\mu\left(E_{i}(\xi)\right)=e_{i}(\xi) \in H^{4 i}(X)$ for $\xi \rightarrow X$ a G-bundle over a finite $C W$-complex.

Let $C^{*}(G) \subset \mathscr{C}^{*}(G)$ be the subalgebra generated by the $E_{i}$. Applying $\mu$, we see that $C^{*}(G)$ is a polynomial algebra on the $E_{i}$. Recall the definition of the completed tensor product $(\$ 2)$.

(4.2) TheOREM. $\mathscr{C}^{*}(G) \approx \Omega_{G}^{*} \hat{\otimes} C^{*}(G)$ additively.

Proof. Since $\Omega_{G}^{*}$ and $C^{*}=C^{*}(G)$ are contained in $\mathscr{C}^{*}=\mathscr{C}^{*}(G)$, there is a map $\rho: \Omega_{G}^{*} \hat{\otimes} C^{*} \rightarrow \mathscr{C}^{*}$ defined on the completed tensor product, given by

$$
\rho\left(\sum n_{i} \otimes c_{i}\right)=\sum n_{i} c_{i} ;
$$

these are infinite sums with the convention (\$2) that the degrees of the $c_{i}$ tend to $+\infty$. In fact, if $\xi$ is a $G$-bundle over a finite complex then $c_{i}(\xi)=0$ for all but a finite number of the $c_{i}$, so $\left(\sum n_{i} c_{i}\right)(\xi)=\sum n_{i} \cdot c_{i}(\xi)$ is essentially a finite sum.

There is a map $\sigma: \mathscr{C}^{*} \rightarrow \lim \operatorname{inv} H^{*}\left(G_{r, s} ; M G\right)(r, s \rightarrow \infty)$, which associates to $\gamma \in \mathscr{C}^{*}$ the element $\left(\gamma\left(\xi_{r, s}\right)\right)$ of the inverse limit; here $\xi_{r, s} \rightarrow G_{r, s}(K)$ is the universal $r$-plane bundle with $K=\boldsymbol{R}, \boldsymbol{C}$ or $\boldsymbol{H}$ depending on $G$. Since the $\xi_{r, s}$ are approximations to universal bundles, it is clear that $\sigma$ is an isomorphism.

In $H^{*}\left(G_{r, s} ; M G\right)$, let $K_{r, s}$ be the subalgebra generated by the cobordism characteristic classes $E_{1}, \ldots, E_{r}$ of the bundle $\xi_{r, s}$. According to $(2.1), H^{*}\left(G_{r, s}\right)$ is 
generated by the cohomology characteristic classes $e_{1}, \ldots, e_{r}$ of $\xi_{r, s}$, and all the relations on the generators are consequences of relations expressed by the Whitney sum formula applied to the Whitney sum $\xi \oplus \eta$. According to (4.1), these same relations hold among the $E_{i}$. Thus the assignment $e_{i} \rightarrow E_{i}$ extends to an algebra homomorphism $\nu: H^{*}\left(G_{r, s}\right) \rightarrow H^{*}\left(G_{r, s} ; M G\right)$ with $\mu \circ \nu=$ identity and

$$
\nu\left\{H^{*}\left(G_{r, s}\right)\right\}=K_{r, s} .
$$

So by (2.2), the natural map $\Omega_{G}^{*} \otimes K_{r, s} \rightarrow H^{*}\left(G_{r, s} ; M G\right)$ is an isomorphism.

By virtue of the naturality of the $E_{i}$, for $r \leqq r^{\prime}$ and $s \leqq s^{\prime}$ the induced map

$$
H^{*}\left(G_{r^{\prime}, s^{\prime}} ; M G\right) \rightarrow H^{*}\left(G_{r, s} ; M G\right)
$$

restricts to a map $K_{r^{\prime}, s^{\prime}} \rightarrow K_{r, s}$. Thus the graded modules $K_{r, s}$ form an inverse system, and the homomorphisms $C^{*} \rightarrow K_{r, s}$ sending $E_{i}$ to $E_{i}\left(\xi_{r, s}\right)$ give rise to a homomorphism $C^{*} \rightarrow \lim$ inv $K_{r, s}$. It follows from (2.1) that this is an isomorphism. We now make use of (2.3) to obtain an isomorphism of $\Omega_{G}^{*} \hat{\otimes} C^{*}$ with

$$
\lim \operatorname{inv} H^{*}\left(G_{r, s} ; M G\right),
$$

which is just the composite $\sigma \circ \rho$. Hence $\rho$ is an isomorphism, as was to be shown.

5. The Hopf algebra $A^{*}(G)$. Under the additive isomorphism $\Psi$ of $\mathscr{A}^{*}(G)$ with $\mathscr{C}^{*}(G)$, there corresponds to $C^{*}(G)$ a graded submodule $A^{*}(G)=\sum_{i \geq 0} A^{i}$ of $\mathscr{A}^{*}(G)$. The elements of $A^{*}(G)$ will be called basic $G$-cobordism operations. Theorem (4.2) has an immediate corollary.

(5.1) THEOREM. $\mathscr{A}^{*}(G) \approx \Omega_{G}^{*} \widehat{\otimes} A^{*}(G)$ additively.

Under this isomorphism $\sum n_{i} \otimes \theta_{i}$ corresponds to $\sum n_{i} \theta_{i}$, with the usual convention concerning infinite sums. In fact, if $\alpha \in H^{*}(X, A ; M G)$ is a cobordism class of a finite $C W$-pair, then $\left(\sum n_{i} \theta_{i}\right) \alpha=\sum n_{i}\left(\theta_{i} \alpha\right)$ is essentially a finite sum since $\theta_{i} \alpha=0$ for almost all $i$.

A basis for $A^{*}(G)$ is obtained as follows. For $\omega=\left(i_{1}, \ldots, i_{r}\right)$ a partition with $i_{1}+\cdots+i_{r}=i$, let $S_{\omega}=S_{\omega}(E)$ be the $S_{\omega}$-symmetric function (or symmetrized monomial; see $[9, \S 1])$ of the $E_{i}$. Thus $S_{\omega} \in C^{d i}(G)$, and as $\omega$ runs through all partitions we obtain a basis for $C^{*}(G)$. The Whitney sum formula for these characteristic classes is

$$
S_{\omega}(\xi \oplus \eta)=\sum_{\omega_{1} \omega_{2}=\omega} S_{\omega_{1}}(\xi) S_{\omega_{2}}(\eta)
$$

We shall identify $A^{*}(G)$ and $C^{*}(G)$ additively, and so $\left\{S_{\omega}\right\}$ is a basis for $A^{*}(G)$.

In order to determine the action on products of cobordism classes, we define a coproduct $\psi^{*}: A^{*} \rightarrow A^{*} \otimes A^{*}$ by

$$
\psi^{*}\left(S_{\omega}\right)=\sum_{\omega_{1} \omega_{1}=\omega} S_{\omega_{1}} \otimes S_{\omega_{2}}
$$

as a map $C^{*} \rightarrow C^{*} \otimes C^{*}$ this is the algebra homomorphism sending $E_{k}$ to

$$
\sum_{i+j=k} E_{i} \otimes E_{j} \text {. }
$$


Let $A^{*} \otimes A^{*}$ act on $H^{*}(X, A ; M G) \otimes H^{*}(Y, B ; M G)$ with values in

by the formula

$$
H^{*}(X \times Y, X \times B \cup A \times Y ; M G)
$$

$$
\left(\sum \theta_{i}^{\prime} \otimes \theta_{i}^{\prime \prime}\right)(\alpha \otimes \beta)=\sum\left(\theta_{i}^{\prime} \alpha\right)\left(\theta_{i}^{\prime \prime} \beta\right) .
$$

(5.2) For $\theta \in A^{*}$ and $\alpha, \beta G$-cobordism classes, $\theta(\alpha \cdot \beta)=\psi^{*}(\theta) \cdot(\alpha \otimes \beta)$.

Proof. It is sufficient to verify this for $\theta=S_{\omega}$ and $\alpha=u_{\xi}, \beta=u_{\eta}$ cobordism Thom classes of $G$-bundles $\xi \rightarrow X$ and $\eta \rightarrow Y$ over finite $C W$-complexes. Let

$$
\xi \times \eta \rightarrow X \times Y
$$

be the Cartesian product of $\xi$ and $\eta$; this is a $G$-bundle with Thom space

$$
M(\xi \times \eta)=M(\xi) \wedge M(\eta)
$$

and $G$-cobordism Thom class $u_{\xi \times \eta}=u_{\xi} \cdot u_{\eta}$ (see [6, §11]). One may now easily check that $S_{\omega}\left(u_{\xi} \cdot u_{\eta}\right)=\psi^{*}\left(S_{\omega}\right) \cdot\left(u_{\xi} \otimes u_{\eta}\right)$ to complete the proof.

In particular, for $X$ a finite complex $H^{*}(X ; M G)$ is a graded algebra over the co-algebras $\left(A^{*}, \psi^{*}\right)$, i.e. (5.2) holds for all $\alpha$ and $\beta$ in $H^{*}(X ; M G)$; see [13]. By an algebra over $A^{*}$ we shall mean, throughout this section, an algebra over the coalgebra $\left(A^{*}, \psi^{*}\right)$.

The rest of this section is devoted to showing that $A^{*}(G)$ is a subalgebra of $\mathscr{A}^{*}(G)$, and that it is in fact a Hopf algebra.

(5.3) THEOREM. $A^{*}(G)$ is a subalgebra of $\mathscr{A}^{*}(G)$.

Assuming this proved, let $\phi^{*}: A^{*} \otimes A^{*} \rightarrow A^{*}$ denote the product homomorphism. It will be left for the reader to convince himself that the coproduct

$$
\psi^{*}: A^{*} \rightarrow A^{*} \otimes A^{*}
$$

is an algebra homomorphism, i.e., to check the formula

$$
\psi^{*}\left(S_{\omega^{\circ}} \circ S_{\omega^{\prime}}\right)(\alpha \otimes \beta)=\left(\psi^{*} S_{\omega} \cdot \psi^{*} S_{\omega^{\prime}}\right)(\alpha \otimes \beta)
$$

for every pair of cobordism classes $\alpha, \beta$. Thus we obtain

(5.4) TheOREM. $\left(A^{*}(G), \phi^{*}, \psi^{*}\right)$ is a graded connected Hopf algebra with commutative coproduct. The ground ring is $Z_{2}$ in the unoriented case and the integers if $\boldsymbol{G}=\boldsymbol{U}$ or $\boldsymbol{S p}$.

Before turning to the proof of (5.3), we describe the action of $A^{*}(G)$ on

$$
H^{*}\left(P^{n}(K) ; M G\right)
$$

this information will also be used in the following section. Let $\xi \rightarrow P^{n}(K)$ be the Hopf $G(1)$-bundle, and put $\alpha=E_{1}(\xi) \in H^{d}\left(P^{n}(K) ; M G\right)$. It follows from (4.1) and (2.2) that as an $\Omega_{G}^{*}$-module, $H^{*}\left(P^{n}(K) ; M G\right)$ is free on the basis $\left\{1, \alpha, \ldots, \alpha^{n}\right\}$ and that $\alpha^{n+1}=0$. Thus it will suffice to determine the action of $S_{\omega}$ on $\alpha$. It is first of all immediate that:

(5.5) If $\xi$ is a $G(1)$-bundle with characteristic class $E_{1}(\xi)$, then the $G$-cobordism classes $S_{\omega}(\xi)$ are given by

$$
S_{k}(\xi)=\left(E_{1}(\xi)\right)^{k}, \quad S_{\omega}(\xi)=0 \quad \text { otherwise. }
$$


(5.6) Lemma. Let $\alpha \in H^{d}\left(P^{n}(K) ; M G\right)$ be the generator. The action of the basic cobordism operations $S_{\omega}$ on $\alpha$ is

$$
S_{k} \alpha=\alpha^{k+1}, \quad S_{\omega} \alpha=0 \text { otherwise. }
$$

Proof. It is well known that the Thom space of the Hopf $G(1)$-bundle $\xi \rightarrow P^{n}(K)$ may be identified with $P^{n+1}(K)$, and that the inclusion of $P^{n}(K)$ in this Thom space is the usual inclusion $P^{n}(K) \subset P^{n+1}(K)$. Thus the Thom class $u \in \bar{H}^{d}\left(P^{n+1}(K) ; M G\right)$ of $\xi$ is carried onto $\alpha=E_{1}(\xi)$ in $H^{d}\left(P^{n}(K) ; M G\right)$. The conclusion now follows directly from (5.5), in view of the isomorphism of $\$ 3$.

(5.7) Corollary. Let $\xi$ be a $G(1)$-bundle. Then $S_{k} \cdot E_{1}(\xi)=\left(E_{1}(\xi)\right)^{k+1}$ and $S_{\omega} \cdot E_{1}(\xi)=0$ otherwise.

Proof of (5.3). Let $\theta, \theta^{\prime} \in A^{*}$ and put $\psi^{*}\left(\theta^{\prime}\right)=\sum \theta_{i}^{\prime} \otimes \theta_{i}^{\prime \prime}$. Let $\gamma$ and $\gamma_{i}^{\prime}$ in $C^{*}$ correspond to $\theta$ and $\theta_{i}^{\prime}$ under the isomorphism $\Psi: C^{*} \approx A^{*}$. If $u_{\xi}$ is the $G$-cobordism Thom class of a $G$-bundle $\xi \rightarrow X$ and $\pi: D(\xi) \rightarrow X$ the associated disk burdle, then $\theta u_{\xi}=u_{\xi} \cdot \pi^{*}(\gamma(\xi))$, and a computation yields

$$
\left(\theta^{\prime} \circ \theta\right) u_{\xi}=u_{\xi} \cdot \pi^{*}\left\{\sum \gamma_{i}^{\prime}(\xi) \cdot \theta_{i}^{\prime \prime} \gamma(\xi)\right\} \text {. }
$$

Thus in order to show that $A^{*}$ is closed under composition it suffices to prove the following statement.

(5.8) If $\theta \in A^{*}(G)$ and $\gamma \in C^{*}(G)$, then there exists $\gamma^{\prime} \in C^{*}(G)$ so that for all $G$-bundles $\xi, \theta \cdot \gamma(\xi)=\gamma^{\prime}(\xi)$.

Moreover, in view of the splitting principle and (2.2) it is enough to prove (5.8) under the assumption that $\xi$ splits into a Whitney sum of $G(1)$-bundles.

Let $R=\boldsymbol{Z}$ if $G=\boldsymbol{U}$ or $\boldsymbol{S p}$ and $\boldsymbol{Z}_{2}$ if $G=\boldsymbol{O}$. Consider a graded polynomial algebra $R\left[\alpha_{1}, \ldots, \alpha_{n}\right]$ on generators of degree $d$, and identify the $G$-cobordism classes $E_{1}, \ldots, E_{n}$ with the elementary symmetric functions of the $\alpha_{i}$. Make $R\left[\alpha_{1}, \ldots, \alpha_{n}\right]$ an algebra over (the coalgebra) $A^{*}(G)$ by requiring that for $i=1, \ldots, n$

$$
S_{k} \cdot \alpha_{i}=\alpha_{i}^{k+1}, \quad S_{\omega} \cdot \alpha_{i}=0 \text { otherwise; }
$$

together with the condition $\theta\left(\beta_{1} \cdot \beta_{2}\right)=\psi^{*} \theta \cdot\left(\beta_{1} \otimes \beta_{2}\right)$ for $\theta \in A^{*}$ and $\beta_{1}, \beta_{2}$ in

$$
R\left[\alpha_{1}, \ldots, \alpha_{n}\right] \text {, }
$$

this specifies the action of $A^{*}$. It is obvious that if $\beta$ is a symmetric polynomial in the $\alpha_{i}$, so is $S_{\omega} \beta$. Thus the subalgebra $R\left[E_{1}, \ldots, E_{n}\right]$ is also an algebra over $A^{*}$.

Now let $\xi=\xi_{1} \oplus \cdots \oplus \xi_{n}$ be a Whitney sum of $G(1)$-bundles over a finite $C W$-complex $X$. We define an algebra homomorphism $f_{\xi}: R\left[\alpha_{1}, \ldots, \alpha_{n}\right] \rightarrow$ $H^{*}(X ; M G)$ by setting $f_{\xi}\left(\alpha_{i}\right)=E_{1}\left(\xi_{i}\right)$. In virtue of (5.7), $f_{\xi}$ is a homomorphism of algebras over $A^{*}$; so is the restriction $f_{\xi}: R\left[E_{1}, \ldots, E_{n}\right] \rightarrow H^{*}(X ; M G)$, and $f_{\xi}\left(E_{i}\right)=E_{i}(\xi)$. It follows that $f_{\xi}(\gamma)=\gamma(\xi)$ for each $\gamma \in R\left[E_{1}, \ldots, E_{n}\right]$. Hence

$$
(\theta \cdot \gamma)(\xi)=\theta \cdot \gamma(\xi)
$$

for $\theta \in A^{*}$ and $\gamma \in R\left[E_{1}, \ldots, E_{n}\right]$. 
It remains to be shown that the actions of $A^{*}$ on $R\left[E_{1}, \ldots, E_{n}\right]$ combine to make $C^{*}=R\left[E_{1}, E_{2}, \ldots\right]$ an algebra over $A^{*}$. For $n \leqq m$, the homomorphism

$$
R\left[\alpha_{1}, \ldots, \alpha_{m}\right] \rightarrow R\left[\alpha_{1}, \ldots, \alpha_{n}\right]
$$

sending $\alpha_{i} \rightarrow \alpha_{i}(i \leqq n)$ and $\alpha_{i} \rightarrow 0(i>n)$ restricts to a homomorphism

$$
R\left[E_{1}, \ldots, E_{m}\right] \rightarrow R\left[E_{1}, \ldots, E_{n}\right]
$$

of algebras over $A^{*}$, sending $E_{i} \rightarrow E_{i}(i \leqq n), E_{i} \rightarrow 0(i>n)$. Taking the inverse limit, we see that $C^{*}$ becomes an algebra over the co-algebra $A^{*}$.

Now let $\theta \in A^{*}$ and $\gamma \in C^{*}$. For $\xi$ a Whitney sum of $n G(1)$-bundles let

$$
\gamma_{1} \in Z_{2}\left[E_{1}, \ldots, E_{n}\right]
$$

be the projection of $\gamma$. Then also $\theta \gamma_{1}$ is the projection of $\theta \gamma$, and so

$$
(\theta \cdot \gamma)(\xi)=\left(\theta \cdot \gamma_{1}\right)(\xi)=\theta \cdot \gamma_{1}(\xi)=\theta \cdot \gamma(\xi) \text {. }
$$

Thus (5.8) is proved for $\xi$ a Whitney sum of $G(1)$-bundles, which completes the proof of Theorem (5.3).

6. Computation of products in $A^{*}(G)$. It was shown in the previous section that $\left(A^{*}(G), \phi^{*}, \psi^{*}\right)$ is a graded connected Hopf algebra with commutative coproduct. The product $\phi^{*}$ is given by the composition of basic $G$-cobordism operations, and $\psi^{*}$ is given in terms of the basis $\left\{S_{\omega}\right\}$ by

$$
\psi^{*} S_{\omega}=\sum_{\omega_{1} \omega_{2}=\omega} S_{\omega_{1}} \otimes S_{\omega_{2}}
$$

The product in $A^{*}(G)$ is complicated, but with the help of the dual Hopf algebra $A_{*}(G)$ and the methods of [11] we can compute products in $A^{*}(G)$.

Let $\left(A_{*}, \psi_{*}, \phi_{*}\right)$ denote the Hopf algebra dual to $A^{*}=A^{*}(G)$. Thus $A_{*}=\sum_{i \geqq 0} A_{i}$ and $A_{i}=\operatorname{Hom}\left(A^{i}, R\right)$, with $R=Z$ for $G=U$ or $S p$ and $R=Z_{2}$ for $G=\boldsymbol{O}$. Let $\left\{\sigma_{\omega}\right\}$ be the basis of $A_{*}$ dual to the basis $\left\{S_{\omega}\right\}$ of $A^{*}$. It is clear that $\psi_{*}\left(\sigma_{\omega} \otimes \sigma_{\omega^{\prime}}\right)=\sigma_{\omega \omega^{\prime}}$, and so $A_{*}$ is a polynomial algebra $R\left[\sigma_{1}, \sigma_{2}, \ldots\right]$ on generators $\sigma_{k}$ of degree $d k$. The coproduct $\phi_{*}: A_{*} \rightarrow A_{*} \otimes A_{*}$ is an algebra homomorphism, so is determined by its values on the algebra generators $\sigma_{k}$. We shall obtain a formula for $\phi_{*}\left(\sigma_{k}\right)$.

In order to state this formula, we introduce some notation. If $\omega=\left(i_{1}, \ldots, i_{r}\right)$ is a partition, let $r_{p}$ denote the number of occurrences of the integer $p$ in $\omega$ and put $R=\left(r_{1}, r_{2}, \ldots\right)$. Thus there is a correspondence $\omega \leftrightarrow R$ between partitions and finitely nonzero sequences of nonnegative integers. We write $S_{\omega}=S^{R}$ and $\sigma_{\omega}=\sigma^{R}$ if $\omega \leftrightarrow R$. Sequences are added component-wise; denote by $\Delta_{k}$ the sequence

$$
\left(r_{1}, r_{2}, \ldots\right)
$$

with $r_{k}=1$ and $r_{p}=0$ for $p \neq k$. In terms of the $S^{R}$, the coproduct of $A^{*}$ is given by

$$
\psi^{*} S_{R}=\sum_{R_{1}+R_{2}=R} S^{R_{1}} \otimes S^{R_{2}}
$$


We make the definitions

$$
\begin{aligned}
\|R\| & =\sum p r_{p}, \quad|R|=\sum r_{p}, \\
\left(\begin{array}{l}
m \\
R
\end{array}\right) & =\frac{m !}{r_{1} ! r_{2} ! \cdots(m-|R|) !} \quad(|R| \leqq m) .
\end{aligned}
$$

Thus $S^{R}$ has degree $d\|R\|$ and $|R|$ is the number of terms of the partition which corresponds to the sequence $R$.

(6.1) THEOREM.

$$
\phi_{*}\left(\sigma_{k}\right)=\sum\left(\begin{array}{c}
m+1 \\
R
\end{array}\right) \sigma_{R} \otimes \sigma_{m}
$$

over all pairs $(R, m)$ with $\|R\|+m=k$ and $|R| \leqq m+1$.

For example, we have

$$
\begin{aligned}
& \phi_{*} \sigma_{1}=\sigma_{1} \otimes 1+1 \otimes \sigma_{1}, \\
& \phi_{*} \sigma_{2}=\sigma_{2} \otimes 1+2 \sigma_{1} \otimes \sigma_{1}+1 \otimes \sigma_{2},
\end{aligned}
$$

hence

$$
\phi_{*}\left(\sigma_{1}\right)^{2}=\left(\sigma_{1}\right)^{2} \otimes 1+2 \sigma_{1} \otimes \sigma_{1}+1 \otimes\left(\sigma_{1}\right)^{2} .
$$

Thus $\sigma_{1}$ and $\sigma_{2}-\left(\sigma_{1}\right)^{2}$ are primitive elements of $A_{*}$ (see $\S 7$ ).

The proof of (6.1) will be preceeded by several lemmas. Let $M^{*}=\sum_{i \geqq 0} M^{i}$ be a graded algebra over the Hopf algebra $A^{*}$, with each $M^{i}$ free and finitely generated over $R$ ( $=Z$ or $Z_{2}$ ). The action of $A^{*}$ on $M^{*}$ induces an action on the graded dual $M_{*}$ of $M^{*}$. Hence there is a homomorphism $M_{*} \otimes A^{*} \rightarrow M_{*}$, which we dualize to obtain a homomorphism

$$
\lambda: M^{*} \rightarrow M^{*} \otimes A_{*} .
$$

The following results may be proved as in [11].

(6.2) Lemma. For $\alpha \in M^{*}$ we have $(\lambda \otimes 1) \lambda(\alpha)=\left(1 \otimes \phi_{*}\right) \lambda(\alpha)$ in $M^{*} \otimes A_{*} \otimes A_{*}$.

(6.3) LEMMA. $\lambda: M^{*} \rightarrow M^{*} \otimes A_{*}$ is an algebra homomorphism.

(6.4) LemMA. If $\lambda(\alpha)=\sum \alpha_{i} \otimes \tau_{i}\left(\alpha, \alpha_{i} \in M^{*}, \tau_{i} \in A_{*}\right)$, then for any $\theta \in A^{*}$

$$
\theta \cdot \alpha=\sum\left\langle\theta, \tau_{i}\right\rangle \alpha_{i} .
$$

Proof of (6.1). Let $\alpha \in H^{d}\left(P^{n}(K) ; M G\right)$ be the canonical generator of

$$
H^{*}\left(P^{n}(K) ; M G\right),
$$

as in $\S 5$. Let $M^{*} C H^{*}\left(P^{n}(K) ; M G\right)$ be the submodule with basis $1, \alpha, \ldots, \alpha^{n}$. By (5.6), $M^{*}$ is an algebra over the Hopf algebra $A^{*}$, and with (6.4) this gives

$$
\lambda \alpha=\alpha \otimes 1+\alpha^{2} \otimes \sigma_{1}+\cdots+\alpha^{n} \otimes \sigma_{n-1} .
$$


Hence

$$
\begin{aligned}
(\lambda \otimes 1) \lambda \alpha & =\sum_{m}(\lambda \alpha)^{m+1} \otimes \sigma_{m} \\
& =\sum_{m}\left\{\sum_{|R| \leqq m+1}\left(\begin{array}{c}
m+1 \\
R
\end{array}\right) \alpha^{\|R\|+m+1} \otimes \sigma^{R}\right\} \otimes \sigma_{m} \\
& =\sum_{k} \alpha^{k-1} \otimes\left\{\sum\left(\begin{array}{c}
m+1 \\
R
\end{array}\right) \sigma^{R} \otimes \sigma_{m}\right\},
\end{aligned}
$$

the final sum over all pairs $(R, m)$ with $\|R\|+m=k$ and $|R| \leqq m+1$. On the other hand,

$$
\left(1 \otimes \phi_{*}\right) \lambda \alpha=\sum_{k} \alpha^{k+1} \otimes \phi_{*} \sigma_{k}
$$

and so for $k<n$ we obtain the desired formula for $\phi_{*}\left(\sigma_{k}\right)$. Letting $n \rightarrow \infty$, the formula holds for all $k$ and the proof is complete.

Notice that $A^{*}(\boldsymbol{U})$ and $A^{*}(\boldsymbol{S p})$ are therefore isomorphic Hopf algebras, although the degrees are doubled. Similarly, $A^{*}(O)$ and $A^{*}(U) \otimes Z_{2}$ are isomorphic Hopf algebras, doubling degrees. In the unoriented case, the binomial coefficients in (6.1) are to be taken mod 2 .

We shall have occasion to use the following computations:

$$
\begin{aligned}
S_{n} \circ S_{n} & =(n+1) S_{2 n}+2 S_{n, n}, \\
S_{m} \circ S_{n} & =(n+1) S_{m+n}+S_{m, n} \quad(m \neq n), \\
S_{2} \circ S_{2 n, 2 n} & \equiv S_{2,2 n, 2 n}+S_{2 n, 2 n+2} \quad(\bmod 2), \\
S_{2 n, 2 n} \circ S_{2} & \equiv S_{2,2 n, 2 n}+S_{2 n, 2 n+2}+S_{4 n+2} \quad(\bmod 2) .
\end{aligned}
$$

For example, consider $S_{2} \circ S_{2 n, 2 n}$. We write $S_{2} \circ S_{2 n, 2 n}=\sum_{\omega} a_{\omega} S_{\omega}$ and must determine the coefficients $a_{\omega}$. Notice that

$$
a_{\omega}=\left\langle S_{2} \otimes S_{2 n, 2 n}, \phi_{*} \sigma_{\omega}\right\rangle .
$$

Now $\phi_{*} \sigma_{2,2 n, 2 n}=\left(\phi_{*} \sigma_{2}\right)\left(\phi_{*} \sigma_{2 n}\right)^{2}$ contains the term

$$
\left(\sigma_{2} \otimes 1\right)\left(1 \otimes \sigma_{2 n}\right)^{2}=\sigma_{2} \otimes \sigma_{2 n, 2 n}
$$

once if $n>1$ and three times if $n=1$; thus $a_{2,2 n, 2 n} \equiv 1(\bmod 2)$. Also $\phi_{*} \sigma_{2 n, 2 n+2}$ $=\left(\phi_{*} \sigma_{2 n}\right)\left(\phi_{*} \sigma_{2 n+2}\right)$ contains the term $\left(1 \otimes \sigma_{2 n}\right)\left(\sigma_{2} \otimes \sigma_{2 n}\right)=\sigma_{2} \otimes \sigma_{2 n, 2 n}$ an odd number of times, namely $2 n+1$, so $a_{2 n, 2 n+2} \equiv 1(\bmod 2)$. It may be seen that $a_{\omega}=0$ for any other $\omega$, so $S_{2} \circ S_{2 n, 2 n}$ is as stated.

7. Generators of $A^{*}(G) \otimes Z_{p}$. Let $A^{*}=A^{*}(G)$ for $G=U$ or $S p$, and let $p$ be a prime. Thus $A^{*} \otimes Z_{p}$ is a Hopf algebra over the field $Z_{p}$, isomorphic to $A^{*}(O)$ if $p=2$. In this section we shall obtain a minimal set of generators of the algebra $A^{*} \otimes Z_{p}$, selected from the basis $\left\{S^{R}\right\}=\left\{S_{\omega}\right\}$.

(7.1) Theorem. A minimal set of generators of $A^{*} \otimes Z_{p}$ is provided by

$$
\left\{S^{p^{k} \Delta_{1}}, S^{p^{k} \Delta_{2}}\right\}_{k \geqq 0}
$$


We remark that if $p$ is odd, another minimal set of generators is $\left\{S^{p^{k} \Delta_{1}}, S^{2 p^{k} \Delta_{1}}\right\}_{k \geqq 0}$. If $p=2$, then among the elements of the basis $\left\{S^{R}\right\}$ only the members of the minimal generating set $\left\{S^{2^{k} \Delta_{1}}, S^{2^{k} \Delta_{2}}\right\}_{k \geqq 0}$ are indecomposable; all others are decomposable in $A^{*} \otimes Z_{2}$.

For the rest of this section we shall fix a prime $p$ and write $A^{*}$ for $A^{*} \otimes Z_{p}$. Thus $A^{*}$ is regarded as a Hopf algebra over $Z_{p}$.

An element $\tau$ of the dual Hopf algebra $A_{*}$ is called primitive if

$$
\phi_{*}(\tau)=\tau \otimes 1+1 \otimes \tau .
$$

For example, it was shown after the statement of (6.1) that $\sigma_{1}$ and $\sigma_{2}-\left(\sigma_{1}\right)^{2}$ are primitive. We denote by $P\left(A_{*}\right)$ the space of primitive elements of $A_{*}$. If $A_{+}^{*}$ denotes the ideal of elements of positive degree in $A^{*}$, then $A_{+}^{*} \cdot A_{+}^{*}$ is the ideal of decomposable elements of $A^{*} . Q\left(A^{*}\right)=A_{+}^{*} / A_{+}^{*} \cdot A_{+}^{*}$ is called the space of indecomposable elements of $A^{*}$. The following results are proved in [13] and [14] respectively.

(7.2) Lemma. $A_{+}^{*} \cdot A_{+}^{*}$ is the annihilator of $P\left(A_{*}\right)$ in $A_{+}^{*}$, hence $P\left(A_{*}\right)$ is canonically isomorphic to the dual of $Q\left(A^{*}\right)$.

(7.3) Lemma. Any set of algebra generators of $A^{*}$ contains a subset whose image in $Q\left(A^{*}\right)$ is a vector space basis; such a subset is minimal and generates $A^{*}$.

As was just mentioned, $\sigma_{1}$ and $\sigma_{2}-\left(\sigma_{1}\right)^{2}$ are primitive elements of $A_{*}$. It follows that for all $k \geqq 0$, the powers $\left(\sigma_{1}\right)^{p^{k}}$ and $\left(\sigma_{2}-\left(\sigma_{1}\right)^{2}\right)^{p^{k}}=\left(\sigma_{2}\right)^{p^{k}}-\left(\sigma_{1}\right)^{2 p^{k}}$ are also primitive. In fact, we shall prove

(7.4) Lemma. $P\left(A_{*}\right)$ has as a basis the set $\left\{\left(\sigma_{1}\right)^{p^{k}},\left(\sigma_{2}\right)^{p^{k}}-\left(\sigma_{1}\right)^{2^{p^{k}}}\right\}_{k \geqq 1}$.

Theorem (7.1) follows directly from these lemmas. In fact, the image of the set $\left\{S^{p^{k} \Delta_{1}}, S^{p^{k} \Delta_{2}}\right\}_{k \geqq 0}$ in $Q\left(A^{*}\right)$ is a vector space basis by virtue of (7.2) and (7.4), so this set is a minimal set of generators of $A^{*}$ according to (7.3). Notice that if $p=2$, $P\left(A_{*}\right)$ also has as basis the set $\left\{\left(\sigma_{1}\right)^{2^{k}},\left(\sigma_{2}\right)^{2^{k}}\right\}$. Thus the remarks following the statement of the theorem follow just as easily.

Thus it remains to prove (7.4). We first show that among the elements $S_{k}$ of $A^{*}$, only $S_{1}$ and $S_{2}$ are indecomposable.

(7.5) Lemma. $S_{k}$ is decomposable if $k>2$.

Proof. To begin with, notice that the computations (6.5) imply that for $m \neq n$,

$$
\left[S_{m}, S_{n}\right]=S_{m} S_{n}-S_{n} S_{m}=(n-m) S_{m+n} .
$$

Suppose first that $p$ is an odd prime. If $k=2 j>2$, then $\left[S_{j-1}, S_{j+1}\right]=2 S_{k}$ so $S_{k}$ is decomposable since $(2, p)=1$. If $k=2 j+1>2$, then $\left[S_{j}, S_{j+1}\right]=S_{k}$ so $S_{k}$ is again decomposable. Now take $k=2$ and consider three cases: $k$ odd, $k \equiv 0(\bmod 4)$ and $k \equiv 2(\bmod 4)$. For $k=2 j+1>2,\left[S_{j}, S_{j+1}\right]=S_{k}$. For $k=4 j>2$, it follows from (6.5) that $S_{2 j} S_{2 j}=S_{k}(\bmod 2)$. Finally for $k=4 j+2>0,(6.5)$ implies that $\left[S_{2 j, 2 j}, S_{2}\right] \equiv S_{k}$ $(\bmod 2)$. Thus the lemma is proved in all cases. 
We now borrow a result from [13]. Let $\xi: A_{*} \rightarrow A_{*}$ be defined by $\xi(a)=a^{p}$; this is a homomorphism of Hopf algebras which multiplies degrees by $p$. There is a natural map $\chi: P\left(A_{*}\right) \rightarrow Q\left(A_{*}\right)$, namely the composition

$$
P\left(A_{*}\right) \rightarrow A_{*}^{+} \rightarrow A_{*}^{+} / A_{*}^{+} \cdot A_{*}^{+} .
$$

Since $A_{*}=Z_{p}\left[\sigma_{1}, \sigma_{2}, \ldots\right], Q\left(A_{*}\right)$ has a basis $\left\{\bar{\sigma}_{k}\right\}$, where $\bar{\sigma}_{k} \in Q\left(A_{*}\right)$ denotes the image of $\sigma_{k} \in A_{*}^{+}$. We remark that $P\left(A_{*}\right)$ is closed under the map $\xi: A_{*} \rightarrow A_{*}$ and that $P\left(\xi A^{*}\right)=\xi P\left(A^{*}\right)$. As a consequence of $[13,(4.21)]$ we have:

(7.6) LEMma. There is an exact sequence

$$
0 \longrightarrow \xi P\left(A_{*}\right) \longrightarrow P\left(A_{*}\right) \stackrel{\chi}{\longrightarrow} Q\left(A_{*}\right) .
$$

We now study the map $\chi: P\left(A_{*}\right) \rightarrow Q\left(A_{*}\right)$. Recall that $Q\left(A_{*}\right)$ has a basis $\left\{\bar{\sigma}_{k}\right\}$; since $\sigma_{1}$ and $\sigma_{2}-\left(\sigma_{1}\right)^{2}$ lie in $P\left(A_{*}\right), \bar{\sigma}_{1}$ and $\bar{\sigma}_{2}$ are in the image of $\chi$.

(7.7) LeMma. For $k>2 \bar{\sigma}_{k}$ is not in the image of $\chi$.

Proof. Suppose $\bar{\sigma}_{k} \in Q\left(A_{*}\right)$ were in the image of $\chi$. Then there would be an element $\tau \in P\left(A_{*}\right)$ of degree $k$ with $\tau \equiv \sigma_{k}$ modulo decomposable elements of $A_{*}$. Now $S_{k} \in A^{*}$ is clearly a primitive element of $A^{*}$, and so $\left\langle S_{k}, \rho\right\rangle=0$ for all decomposable elements $\rho$ of $A_{*}$ by (7.2). Thus $\left\langle S_{k}, \tau\right)=\left\langle S_{k}, \sigma_{k}\right\rangle=1$. But if $k>2$ (7.5) states that $S_{k}$ is decomposable, and so by (7.2) again $\left\langle S_{k}, \tau\right\rangle=0$ since $\tau \in P\left(A_{*}\right)$. This is a contradiction, which completes the proof.

Proof of (7.4). We write $P=P\left(A_{*}\right)$ and let $P^{\prime} C P$ be the subspace with $\sigma_{1}$ and $\sigma_{2}-\left(\sigma_{1}\right)^{2}$ as basis. It follows from (7.6) and (7.7) that $P=\xi P \oplus P^{\prime}$. We may apply $\xi$ repeatedly to obtain for $k \geqq 0$

$$
P=\xi^{k+1} P \oplus\left(\xi^{k} P^{\prime} \oplus \cdots \oplus \xi P^{\prime}+P^{\prime}\right) .
$$

Since $\bigcap_{k} \xi^{k} P=(0)$, it follows that $P=\bigoplus_{k=0}^{\infty} \xi^{k} P^{\prime}$. Now $\xi^{k} \sigma_{1}=\left(\sigma_{1}\right)^{p^{k}}$ and

$$
\xi^{k}\left(\sigma_{2}-\left(\sigma_{1}\right)^{2}\right)=\left(\sigma_{2}\right)^{p^{k}}-\left(\sigma_{1}\right)^{2 p^{k}},
$$

hence the lemma, and with it Theorem (7.1), is proved.

8. Subalgebras of $A^{*}(G) \otimes Z_{p}$. In this section we shall show that the Hopf algebras $A^{*}(G) \otimes Z_{p}, G=U$ or $S p$, and $A^{*}(O)$ contain as Hopf subalgebras suitable quotients of the mod $p$ Steenrod algebras $\mathscr{S}^{*}(p)$. In addition, we determine the primitively generated subalgebras of $A^{*}(G) \otimes Z_{p}$ and $A^{*}(O)$.

(8.1) THEOREM. There are degree-preserving homomorphisms of Hopf algebras $\Gamma: \mathscr{S}^{*}(p) \rightarrow A^{*}(G) \otimes Z_{p}$ for $G=U$ or $S p$, and $\Gamma: \mathscr{S}^{*}(2) \rightarrow A^{*}(0)$, such that for $\theta \in \mathscr{S}^{*}(p)$ and $\theta^{\prime} \in A^{*}(G)$ the diagrams

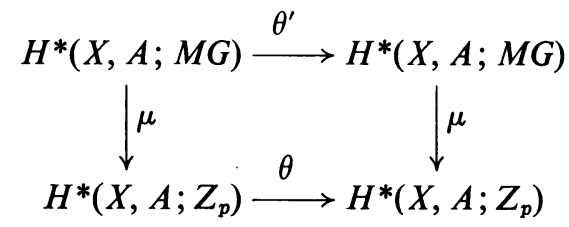

are commutative for all finite $C W$-pairs if and only if $\Gamma(\theta)=\theta^{\prime} \otimes 1$. 
Let $\left(\beta_{p}\right)$ denote the two-sided ideal generated by the Bockstein in $\mathscr{S}^{*}(p)$. Let $\xi: \mathscr{S}^{*}(2) \rightarrow \mathscr{S}^{*}(2)$ be the dual of the squaring map in the dual Hopf algebra $\mathscr{S}_{*}(2)$. Thus $\xi$ and $\xi^{2}=\xi \circ \xi$ are Hopf algebra homomorphisms of $\mathscr{S}^{*}(2)$ into itself, so their kernels are Hopf ideals in $\mathscr{S}^{*}(2)$ (see Liulevicius [10]). Notice that $\beta_{2}=S q^{1}$ and $\left(\beta_{2}\right)=\operatorname{ker}(\xi)$ in $\mathscr{S}^{*}(2)$, by Milnor [12].

(8.2) THEOREM. The kernel of $\Gamma$ for $G=U$ or $S p$ and $p$ an odd prime is exactly $\left(\beta_{p}\right)$. For $p=2$ and $G=\boldsymbol{O}, U$ or $S p$, the kernel of $\Gamma$ is $(0), \operatorname{ker}(\xi)$ or $\operatorname{ker}\left(\xi^{2}\right)$ respectively.

It is convenient to introduce the stable operations from $G$-cobordism to cohomology. Let $R=Z$ or $Z_{p}, p$ a prime, for $G=U$ or $S p$, and $R=Z_{2}$ if $G=O$. We denote by $\mathscr{A}^{i}(G ; R)$ the set of linear operations

$$
\nu: H^{k}(X, A ; M G) \rightarrow H^{k+i}(X, A ; R)
$$

defined for all finite $C W$-pairs, which commute with induced maps and suspensions. Under addition of operations, $\mathscr{A}^{*}(G ; R)=\sum_{i \geqq 0} \mathscr{A}^{t}(G ; R)$ is a graded $R$-module. Using finite approximations to the Thom spaces $M G(n)$ as in $\S 4$, it is easily shown that $\mathscr{A}^{*}(G ; R)$ is naturally isomorphic to $H^{*}(M G ; R)$. Moreover, there is an isomorphism $\Psi: \mathscr{A}^{*}(G ; R) \approx R\left[e_{1}, e_{2}, \ldots\right]$ entirely analogous to the isomorphism $\Psi$ of $\S 3$ (see the concluding remarks of $\S 3$ ).

There is a linear degree-preserving map $\mu^{L}: A^{*}(G) \rightarrow \mathscr{A}^{*}(G ; R)$ defined as follows. Let $\mu: H^{*}(; M G) \rightarrow H^{*}(; R)$ be the natural transformation. For $\theta \in A^{*}(G)$, put $\mu^{L}(\theta)=\mu \circ \theta$, composition with $\mu$ on the left. It is clear that there is a commutative diagram

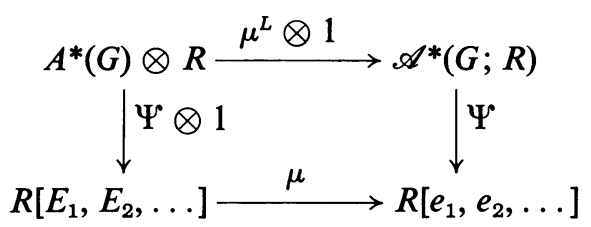

hence,

(8.3) LemMA. $\mu^{L} \otimes 1: A^{*}(G) \otimes R \rightarrow \mathscr{A}^{*}(G ; R)$ is an additive isomorphism.

In addition, there are linear maps $\mu^{R}: \mathscr{S}^{*}(p) \rightarrow \mathscr{A}^{*}\left(G ; Z_{p}\right)$, defined by putting $\mu^{R}(\theta)=\theta \circ \mu$ for $\theta \in \mathscr{S}^{*}(p)(p=2$ if $G=O)$. In fact, there is a map

$$
\mathscr{S}^{*}(p) \otimes \mathscr{A}^{*}\left(G ; Z_{p}\right) \rightarrow \mathscr{A}^{*}\left(G ; Z_{p}\right)
$$

sending $\theta \otimes \nu$ to the composition $\theta \circ \nu$, thereby making $\mathscr{A}^{*}\left(G, Z_{p}\right)$ a graded module over $\mathscr{S}^{*}(p)$. Since $\mu \in \mathscr{A}^{*}\left(G ; Z_{p}\right)$, the homomorphism $\mu^{R}$ is given by the action of $\mathscr{S}^{*}(p)$ on $\mu$. Under the natural isomorphism of $\mathscr{A}^{*}\left(G ; Z_{p}\right)$ with $H^{*}\left(M G ; Z_{p}\right), \mu$ corresponds to the "unit class" in $H^{*}\left(M G ; Z_{p}\right)$, and the action (by composition) of $\mathscr{S}^{*}(p)$ on $\mathscr{A}^{*}\left(G ; Z_{p}\right)$ is seen to correspond to the usual module structure of $H^{*}\left(M G ; Z_{p}\right)$ over $\mathscr{S}^{*}(p)$. 
Proof of (8.1). Let $\Gamma: \mathscr{S}^{*}(p) \rightarrow A^{*}(G) \otimes Z_{p}$ denote the composition

$$
\left(\mu^{L} \otimes 1\right)^{-1} \circ \mu^{R} \text {. }
$$

Thus $\Gamma$ is linear and degree-preserving, and for $\theta \in \mathscr{S}^{*}(p)$ we have $\left(\mu^{L} \otimes 1\right) \Gamma(\theta)$ $=\mu^{R}(\theta)$. In view of the definitions of $\mu^{L}$ and $\mu^{R}$, for $\theta^{\prime} \in A^{*}(G)$ we have $\Gamma(\theta)=$ $\theta^{\prime} \otimes 1$ if and only if $\mu \circ \theta^{\prime}=\theta \circ \mu$.

To show that $\Gamma$ is an algebra homomorphism, let $\theta_{1}, \theta_{2} \in \mathscr{S}^{*}(p)$ with $\Gamma\left(\theta_{1}\right)$ $=\theta_{1}^{\prime} \otimes 1$ and $\Gamma\left(\theta_{2}\right)=\theta_{2}^{\prime} \otimes 1$. Then $\mu \circ \theta_{i}^{\prime}=\theta_{i} \circ \mu(i=1,2)$, from which it follows that $\mu \circ\left(\theta_{1}^{\prime} \circ \theta_{2}^{\prime}\right)=\left(\theta_{1} \circ \theta_{2}\right) \circ \mu$, hence $\Gamma\left(\theta_{1} \circ \theta_{2}\right)=\Gamma\left(\theta_{1}\right) \Gamma\left(\theta_{2}\right)$. In a more tedious fashion, it may be shown that $\Gamma$ commutes with the coproducts; recall that the coproduct in $\mathscr{S}^{*}(p)$ satisfies an analogue of (5.2). Thus $\Gamma$ is a homomorphism of Hopf algebras.

Proof of (8.2). From the definition of $\Gamma$, we see that it has the same kernel as $\mu^{R}: \mathscr{S}^{*}(p) \rightarrow \mathscr{A}^{*}\left(G ; Z_{p}\right)$. Hence $\operatorname{ker}(\Gamma)$ is the annihilator in the Steenrod algebra $\mathscr{S}^{*}(p)$ of the unit class in $H^{*}\left(M G ; Z_{p}\right)$. Thus (8.2) follows from results of Milnor [12]; for $p=2$ see Liulevicius [10].

REMARK. Unless $G=S p$ and $p$ is odd, the image of $\Gamma$ in $A^{*}(G) \otimes Z_{p}$ has as a $Z_{p}$-basis the elements $S_{\omega}$ for $\omega$ a partition involving only integers of the form $p^{k}-1$.

For example, let $\boldsymbol{G}=\boldsymbol{U}$. Milnor [12] has shown, in slightly different notation, that $\mathscr{S}^{*}(p) /\left(\beta_{p}\right)$ has a basis $\left\{t_{\omega}\right\}$ over $Z_{p}, \omega=\left(i_{1}, \ldots, i_{r}\right)$ running through partitions involving only integers of the form $p^{k}-1$, such that

(a) $t_{\omega}$ has degrees $2\left(i_{1}+\cdots+i_{r}\right)$;

(b) the coproduct sends $t_{\omega}$ to $\sum_{\omega_{1} \omega_{2}=\omega} t_{\omega_{1}} \otimes t_{\omega_{2}}$;

(c) if $x \in H^{2}\left(X ; Z_{p}\right)$, then $t_{p^{k}-1} x=x^{p^{k}}$ and $t_{\omega} x=0$ otherwise.

Thus if $\alpha \in H^{2}\left(P^{n}(C) ; M U\right)$ is the generator and $\omega$ involves only integers of the form $p^{k}-1, \mu\left(S_{\omega}(\alpha)\right)=t_{\omega} \mu(\alpha)$ in $H^{*}\left(P^{n}(C) ; Z_{p}\right)$. It follows easily from the splitting principle and universality that $\mu\left(S_{\omega}\left(u_{\xi}\right)\right)=t_{\omega} \mu\left(u_{\xi}\right)$ whenever $u_{\xi}$ is the $U$-cobordism Thom class of a $U$-bundle $\xi \rightarrow X$ over a finite complex, hence

$$
\mu \circ S_{\omega}=t_{\omega} \circ \mu \in \mathscr{A}^{*}\left(U ; Z_{p}\right) .
$$

But this says exactly that $\Gamma\left(t_{\omega}\right)=S_{\omega}$, verifying the statement.

Let $A^{*}=A^{*}(G) \otimes Z_{p}$ for fixed $G$ and $p(p=2$ if $G=O)$. The nonzero primitive elements of $A^{*}$ are evidently the $S_{k}$ for $k>0$. Let $B^{*}$ denote the subalgebra generated by these elements and the identity. In fact, since the $S_{k}$ are primitive, $B^{*}$ is a Hopf subalgebra of $A^{*}$.

(8.4) THEOREM. A basis for $B^{*}$ consists of the elements $S_{\omega}$ for which no integer has $\geqq p$ occurrences in $\omega$.

Proof. Let $\bar{B}^{*}$ be the submodule of $A^{*}$ with the basis described in the theorem. We first show that $\bar{B}^{*}$ is a Hopf subalgebra of $A^{*}$. It is clear that $\psi^{*}\left(\bar{B}^{*}\right) \subset \bar{B}^{*} \otimes \bar{B}^{*}$. 
The annihilator of $\bar{B}^{*}$ in the dual Hopf algebra $A_{*}=Z_{p}\left[\sigma_{1}, \sigma_{2}, \ldots\right]$ is the ideal $I_{*}$ generated by $\sigma_{1}^{p}, \sigma_{2}^{p}, \ldots$. It follows from (6.1) that

$$
\phi_{*}\left(\sigma_{k}^{p}\right)=\sum\left(\begin{array}{c}
m+1 \\
R
\end{array}\right)\left(\sigma^{R}\right)^{p} \otimes \sigma_{m}^{p}
$$

summed over certain pairs $(R, m)$; hence $\phi_{*}\left(\sigma_{k}^{p}\right) \in I_{*} \otimes A_{*}+A_{*} \otimes I_{*}$. Thus

$$
\phi_{*}\left(I_{*}\right) \subset I_{*} \otimes A_{*}+A_{*} \otimes I_{*},
$$

which means that $\bar{B}^{*}$ is a subalgebra of $A^{*}$. Since $S_{k} \in \bar{B}^{*}$ for all $k, B^{*} \subset \bar{B}^{*}$. Hence it remains to show that $\bar{B}^{*} \subset B^{*}$.

We shall show that each basis element $S_{i_{1}, i_{2}, \ldots, i_{r}} \in \bar{B}^{*}$ belongs to $B^{*}$ by induction on the number of terms $r$. If $r=1$, then $S_{i_{1}}$ is a generator of $B^{*}$. If $r>1$, suppose that $i_{1} \leqq i_{2} \leqq \cdots \leqq i_{r}$ and notice that the number $m$ of occurrences of $i_{r}$ is less than $p$. A judicious application of (6.1) now shows that

$$
S_{i_{r}} \circ S_{i_{1}, \ldots, i_{r-1}}=m S_{i_{1}, \ldots, i_{r}}+\sum a_{\omega} S_{\omega}, a_{\omega} \in Z_{p},
$$

with the sum over partitions of the form

$$
\omega=\left(i_{1}, \ldots, i_{n-1}, i_{n+1}, \ldots, i_{r-1}, i_{n}+i_{r}\right),
$$

for $1 \leqq n \leqq r-1$, having $(r-1)$ terms. Since $m$ is prime to $p$ and the basis elements $S_{i_{1}, \ldots, i_{r-1}}$ and the $S_{\omega}$ of the sum belong to $\bar{B}^{*}$, so to $B^{*}$ by hypothesis of the induction, the proof is complete.

9. Operations on the bordism rings. The coefficient ring $\Omega_{G}^{*}=H^{*}(p t ; M G)$ of the $G$-cobordism theory is a graded module over the Hopf algebra $A^{*}(G)$. Recall that $\Omega_{G}^{-n}=\pi_{d k+n}(M G(k))$ for $k$ large; in particular $\Omega_{G}^{n}=0$ for $n>0$. Since the bordism group $\Omega_{n}^{G}$ of $n$-dimensional $G$-manifolds is also naturally isomorphic to this stable homotopy group, we may identify $\Omega_{n}^{G}$ with $\Omega_{G}^{-n}$ for $n \geqq 0$. There results an action

$$
A^{i}(G) \otimes \Omega_{n}^{G} \rightarrow \Omega_{n-i}^{G}
$$

on the $G$-bordism ring. Thus $\theta\left(\theta^{\prime}[M]\right)=\left(\theta \circ \theta^{\prime}\right)[M]$ and if $\psi^{*}(\theta)=\sum \theta_{i}^{\prime} \otimes \theta_{i}^{\prime \prime}$ then $\theta([M] \cdot[N])=\sum\left(\theta_{i}^{\prime}[M]\right)\left(\theta_{i}^{\prime \prime}[N]\right)$.

REMARK. This action may also be given the following description; the proof of the equivalence of the two definitions is omitted. Let $M^{n}$ be an $n$-dimensional $G$-manifold and let $\theta \in A^{i}(G)$ correspond to the $G$-cobordism characteristic class $\gamma$. Thus if $\xi \rightarrow M^{n}$ denotes the stable normal $G$-bundle of $M, \gamma(\xi) \in H^{i}\left(M^{n} ; M G\right)$. By Poincaré duality $[6, \S 13 ; 16]$ there results a $G$-bordism class in $H_{n-i}\left(M^{n} ; M G\right)$; such a class has a representative $\left(V^{n-i}, f\right)$ consisting of a $G$-manifold $V^{n-i}$ and a continuous map $f: V^{n-i} \rightarrow M^{n}$. Put $\theta\left[M^{n}\right]=\left[V^{n-i}\right]$.

The remainder of the discussion will be restricted to the complex case. If $\left[M^{2 n}\right] \in \Omega_{2 n}^{U}$ and $\theta \in A^{2 n}(U)$, the result of the action is $\theta\left[M^{2 n}\right] \in \Omega_{0}^{U}=Z$. Thus we obtain characteristic numbers of the bordism class, which may be easily identified 
with the dual Chern numbers. Hence there is a homomorphism $\Delta: \Omega_{2 n}^{U} \rightarrow A_{2 n}(\boldsymbol{U})$ so that $\left\langle\theta, \Delta\left[M^{2 n}\right]\right\rangle=\theta\left[M^{2 n}\right]$ for all $\theta \in A^{2 n}(U)$. In fact, $\Delta: \Omega_{*}^{U} \rightarrow A_{*}(\boldsymbol{U})$ is an algebra homomorphism into the dual of $A^{*}(U)$, by virtue of the coproduct formula for $\theta([M] \cdot[N])$.

Now fix a prime $p$ and put $A^{*}=A^{*}(\boldsymbol{U}) \otimes Z_{p}$. Let $S^{*}$ denote the image of $\Gamma$ in $A^{*}$. There is a ring homomorphism $\Delta \otimes 1: \Omega_{*}^{U} \otimes Z_{p} \rightarrow A_{*}(\boldsymbol{U}) \otimes Z_{p}=A_{*}$, whose image we shall study. The following result is due to Atiyah and Hirzebruch [1, §5], and is essentially the determination of the relations on $\bmod p$ Chern numbers.

(9.1) THEOREM. The image of $\Delta \otimes 1$ in $A_{*}$ has for annihilator in $A_{*}$ the right ideal $S_{+}^{*} A^{*}$ generated by the set $S_{+}^{*}$ of elements of $S^{*}$ with positive degree.

Proof. Let $\theta \in \mathscr{S}^{2 n}(p), n>0$, so that $\Gamma(\theta)=\theta^{\prime} \otimes 1$ with $\theta^{\prime} \in A^{2 n}(U)$. By (8.1), if $\left[M^{2 n}\right] \in \Omega_{2 n}^{U} \approx \Omega_{U}^{-2 n}$ then $\mu \theta^{\prime}\left[M^{2 n}\right]=\theta \mu\left[M^{2 n}\right]=0$ since $\mu\left[M^{2 n}\right] \in H^{-2 n}(p t ; Z p)$. Thus $\theta^{\prime}\left[M^{2 n}\right]$ is divisible by $p$, so $\left\langle\Gamma(\theta), \Delta\left[M^{2 n}\right] \otimes 1\right\rangle=\Gamma(\theta)\left(\left[M^{2 n}\right] \otimes 1\right)=0$. Hence $S_{+}^{*}$ annihilates the image of $\Delta \otimes 1$, and so does $S_{+}^{*} A^{*}$ since the annihilator is easily seen to be a right ideal in $A^{*}$. By Milnor [12], the image of $\Delta \otimes 1$ in $A_{*} \approx H_{*}\left(\boldsymbol{M U} ; Z_{p}\right)$ is a polynomial algebra over $Z_{p}$ with one generator in each dimension $2 k$ not of the form $2 p^{i}-2$; moreover, $A^{*} \approx H^{*}\left(\boldsymbol{M U} ; Z_{p}\right)$ is a free left module over

$$
S^{*} \approx \mathscr{S}^{*}(p) /\left(\beta_{p}\right),
$$

with a basis consisting of the $S_{\omega}$ for which $\omega$ involves no integers of the form $p^{i}-1$. A dimension count shows that $S_{+}^{*} A^{*}$ is exactly the annihilator of the image of $\Delta \otimes 1$.

REMARK. In the unoriented case, put $I^{*}=\sum I^{n}$ with $I^{n}$ consisting of all $\theta \in A^{n}(O)$ for which $\theta\left[M^{n}\right]=0 \forall\left[M^{n}\right] \in \Omega_{n}^{o}$. It is easily seen that $I^{*}$ is a right ideal in $A^{*}(O)$, and the argument just given shows that $I^{*}$ is the right ideal generated by the elements of the image of $\Gamma: \mathscr{S}^{*}(2) \rightarrow A^{*}(\boldsymbol{O})$ of positive degree. In the symplectic case, there is an analogue of (9.1) for $p$ odd but not for $p=2$.

Conjecture. The right ideal $S_{+}^{*} A^{*}$ in $A^{*}=A^{*}(\boldsymbol{U}) \otimes Z_{p}$ (see (9.1)) contains no nontrivial left ideals. The analogous assertion in the unoriented case implies that the action of $A^{*}(\boldsymbol{O})$ on $\Omega_{*}^{\boldsymbol{O}}$ is effective, i.e., if $\theta \in A^{*}(\boldsymbol{O})$ satisfies $\theta[M]=0 \forall[M]$ $\in \Omega_{*}^{o}$ then $\theta=0$.

Added in proof. The present paper has considerable overlap with recent work of S. P. Novikov and J. M. Boardman. Novikov studies the Adams spectral sequence for $U$-cobordism; an announcement has appeared in Dokl. Akad. Nauk SSSR 172 (1967), 33-36. Boardman studies the nonoriented case, with applications to smooth involutions; see Bull. Amer. Math. Soc. 73 (1967), 136-138, and Chapter VI of his Warwick notes "Stable Homotopy Theory."

\section{REFERENCES}

1. M. F. Atiyah and F. Hirzebruch, Cohomologie-Operationen und characteristische Klassen, Math. Z. 77 (1961), 149-187.

2. A. Borel, La cohomologie mod 2 de certains espaces homogènes, Comment. Math. Helv. 27 (1953), 165-197. 
3. - Sur la cohomologie des espaces fibrés principaux et des espaces homogènes des groupes de Lie compacts, Ann. of Math. 57 (1953), 115-207.

4. A. Borel and F. Hirzebruch, Characteristic classes and homogeneous spaces. I, Amer. J. Math. 80 (1958), 458-538.

5. P. E. Conner and E. E. Floyd, Cobordism theories, Seattle Topology Conference, 1963 (mimeographed).

6. —_ Differentiable periodic maps, Ergebnisse der Math., Vol. 33, Springer-Verlag, Berlin, 1964.

7. - Relations between cobordism and K-theory, Springer Lecture Notes in Mathematics, 1967.

8. A. Dold, Relations between ordinary and extraordinary cohomology, Colloquium on Algebraic Topology, Aarhus Universitet, 1962, pp. 1-9.

9. F. Hirzebruch, Neue topologische Methoden in der algebraischen Geometrie, Ergebnisse der Math., Vol. 9, Springer-Verlag, Berlin, 1956.

10. A Liulevicius, Notes on homotopy of Thom spectra, Amer. J. Math. 86 (1964), 1-16.

11. J. Milnor, The Steenrod algebra and its dual, Ann. of Math. 67 (1958), 150-171.

12. - On the cobordism ring $\Omega^{*}$ and a complex analogue, Amer. J. Math. 82 (1960), 505-521.

13. J. Milnor and J. C. Moore, On the structure of Hopf algebras, Ann. of Math. 81 (1965), 211-264.

14. N. W. Steenrod and D. Epstein, Cohomology operations, Annals of Mathematics Studies, No. 50, Princeton Univ. Press, Princeton, N. J., 1962.

15. R. Thom, Quelques propriétés globales des variétés différentiables, Comment. Math. Helv. 28 (1954), 17-86.

16. G. W. Whitehead, Generalized homology theories, Trans. Amer. Math. Soc. 102 (1962), 227-283.

UNIVERSITY OF VIRGINIA,

Charlottesville, Virginia 